\title{
Acceleration of Born Series by change of variables
}

\author{
Hector Lopez-Menchon, Juan M. Rius, Senior Member, IEEE, Alexander Heldring, and Eduard Ubeda,
}

\begin{abstract}
In this work, we propose a method to enhance the convergence of the Born series. The Born series is widely used in scattering theory, but its convergence is only guaranteed under certain restrictive conditions which limit the cases where this formulation can be applied. The proposed method, based on modifying the singularities of the resolvent operator by a change of variables, accelerates the convergence of the series or even achieves convergence in otherwise divergent cases. Due to its low computational cost and ease of implementation, the method preserves the advantages of the Born series while it extends its range of application. It can also be applied to a variety of methods relying on the same mathematical principles as the Born series. Numerical examples are provided to show that a significant improvement can be achieved with simple techniques and with a limited knowledge about the operator spectrum.
\end{abstract}

Index Terms-Born series, convergence acceleration, domain decomposition methods, fixed point methods, numerical methods, numerical simulation.

\section{INTRODUCTION}

$\mathbf{T}$ HE Born series is a classical technique for solving scattering problems, which was introduced by Max Born in the 1920s as a method for solving the Lippmann-Schwinger equation in the framework of quantum theory of scattering [1]. The Born series is in fact equivalent to the Liouville-Neumann series (or simply Neumann series), which was independently proposed by Liouville and Neumann in the 19th century as a method for solving a certain kind of functional equations.

The Neumann series expands the inverse of a linear operator as an operator power series and is of great importance in Fredholm theory [2]. It is closely related to fixed-point iteration methods [3]. These methods, aimed at solving equations of the form $x=f(x)$, start from an initial $x_{0}$ and refine the solution by repeatedly applying the recurrence relation $x_{k}=f\left(x_{k-1}\right)$. The Neumann series can be efficiently written as a fixed point iteration method, and this double approach will be present throughout this text. The main drawback of the Neumann series is that convergence is only guaranteed under some restrictive spectral conditions [4].

In scattering theory, the best known fixed-point iteration method is the Born iteration or its equivalent, the Born series. A large variety of methods are based on the same principles as the Born series, although this connection sometimes does not explicitly appear in the literature. These methods differ in the formalism used to model the scattering phenomenon,

Manuscript received June, 30th, 2020. This work was partly funded by the Ministerio de Ciencia e Innovacion (MICINN) under projects TEC201678028-C3-1-P, MDM2016-0600, TEC2017-83343-C4-2-R, TEC2017-84817C2-2-R, PID 2019-107885GB-C31 and Catalan Research Group 2017 SGR 219; and by Secretary of Universities and Research of the Ministry of Business and Knowledge of the Generalitat of Catalonia and European Social Fund under scholarship 2019 FI_B00788.

The authors are with CommSensLab, Universitat Politecnica de Catalunya (BarcelonaTECH), Barcelona, Spain, e-mail: hector.lopez.menchon@upc.edu. and their common feature is the iterative procedure used to solve the overall equation. Below, we give an overview and succinctly describe a few variations of the Born series.

The Born series is used to solve scattering problems in several contexts such as quantum mechanics, acoustics, or seismics. Here, we will focus exclusively on the wave equation of classical electromagnetics. The most common form of the Born series consists of a Neumann series directly built on the Volume Integral Equation [5]. One of its variations is the Generalized Born Series [6], [7], which is usually applied to sets of several scatterers. The local scattering problems associated to each object are solved by an arbitrary method (BIE, FDTD, analytic methods or even Born Series itself), and the global problem involving interactions between objects is solved by the Born series, so that the generalized Born series can be thought of as a domain decomposition method, as in [8].

The Neumann series is also used in the framework of radiative transfer [9, p. 65]. In this case, ensembles of a very large number of particles are considered, and each particle is assumed to be in the far field region of all the other ones [10]. Then, the local scattering problem is solved by a farfield analytic method, and the overall field is computed by an iterative method usually referred to as order of scattering expansion [10], multiple scattering [11], or successive order of interaction [12]. This method can be recognized as a Neumman series applied to the so-called far-field Foldy-Lax equations [9].

Iterative Physical Optics (IPO) [13]-[15] recursively applies the Physical Optics approximation to account for highfrequency multiple interactions in many interesting problems, like the computation of Radar Cross Section (RCS) from large targets, or antennas radiating in complex environments. Repeated computation of the multiply-scattered field is equivalent to a Neumann series or fixed-point iteration.

All the methods above rely on the same fixed-point iteration or Neumann series scheme. As we have mentioned, they may suffer from convergence problems. The objective of this work is to implement a general and simple technique to enhance the convergence of this kind of methods. For this purpose, we will resort to series acceleration methods [16], which have been widely used to improve the convergence of sequences in numerical computations. Although they are frequently used in scalar sequences, series acceleration methods can also be applied to vector and matrix cases, giving rise to extrapolation methods [17], like the well known Richardson's method [18, p. 136]. Some acceleration methods have already been applied to fixed-point schemes in seismic [19] and electromagnetic [20] scattering. However, such implementations require, at each iteration, vector operations involving several terms of the original sequence. This, besides its computational cost, 
moves away from the simplicity of fixed-point methods. Other acceleration methods applied to scattering do not use this multistep approach, but also require complex operations at each iteration [21] or are targeted at a specific problem [22]. Our method, in contrast, only involves a set of scalar coefficients multiplying the terms of the original series, thus keeping the simplicity of the original method. Computing these coefficients is inexpensive as it does not require matrix or vector operations. Moreover, since our transformation depends on an arbitrary change of variable, the method can be easily adapted to different kinds of problems.

Another method to improve the convergence of the Born series consists of including a multiplicative parameter in the simple iteration, which is equivalent to a scalar preconditioning [23]-[25]. Although this method may provide excellent results in some situations, it is limited to the cases where the convex envelope of the operator spectrum excludes the origin of the complex plane.

The objective of this work is to introduce the mathematical foundations of Born series acceleration by a change of variables. The proposed technique is illustrated with very simple examples. More complex and powerful changes of variable and the necessary techniques for adaptively tuning their parameters will be addressed in a forthcoming publication.

This paper is organized as follows: in Section II we theoretically describe our method. The foundations of Neumann series and resolvent formalism are introduced (II-A), and our acceleration method is described (II-B). Finally, we arrive at the final form of our method (II-C), and state some considerations about its application to electromagnetic scattering (II-D). Sections III and IV present some numerical examples and the paper conclusions, respectively.

\section{THEORY}

\section{A. Neumann series and Taylor expansion of the resolvent}

In this section we will give a short description of the Neumann series methods and their connection to the Born series. A Neumann series expresses the inverse of an operator as an operator power series.

Let us consider a linear functional equation

$$
u=H u+b,
$$

which corresponds to the form $u=f(u)$ at which fixed point methods are aimed. In general, $u$ is the unknown function, $b$ is a known function and $H$ is a linear operator defined in some functional space, that will be assumed to be a Hilbert space. The MFIE for conductors, for example, is an equation of this kind, in which the unknown function is the electric current, the independent function is linked to the incident field and $H$ is an integro-differential operator. It is a Fredholm equation of the second kind.

For the sake of simplicity, we will work with the discretized operator and assume that $u$ and $b$ are vectors of $\mathbb{C}^{n}$ and $H$ is a complex matrix. However, the following derivations can be easily generalized for other vector spaces.
A way to solve equations of the form (1) under certain conditions is the classical fixed-point iteration

$$
u_{n}=H u_{n-1}+b
$$

with $u_{0}=b$. This method is the basis of several classical stationary methods such as Jacobi and Gauss-Seidel. In this context, the $H$ matrix is known as the iteration matrix. If $H$ fulfills certain spectral conditions, this series will converge towards the solution, $\lim _{n \rightarrow \infty} u_{n}=u$.

In the infinite limit, the series in (2) is mathematically equivalent to the power expansion

$$
u=(I-H)^{-1} b=\sum_{k=0}^{\infty} H^{k} b,
$$

where $I$ is the identity operator. This formalism is known as the Neumann series. The approximate solution $u_{n}$ can be obtained from (3) by truncating the series at the $n$-th element. Although these expressions are equivalent, the iterative form (2) is more suitable than (3) for computational implementation, whereas (3) may provide more mathematical insight.

Unfortunately, the convergence of this series is not guaranteed. The convergence condition for (3) is expressed in terms of the spectral radius of $H$. The spectral radius $\rho$ of a matrix is defined as the maximum of the modulus of the eigenvalues $\lambda_{i}$ of $H$, that is $\rho(H)=\max _{i}\left|\lambda_{i}\right|$. It is known that the iteration (2), as well as the series in (3), converges if the spectral radius of the iteration matrix is smaller than one, $\rho(H)<1$ [18].

This convergence condition is quite restrictive, and most of the systems of interest do not fulfill it. However, there are methods to achieve or accelerate convergence. In order to apply these methods, it is convenient to write the series in a suitable way. The first step is to expand the solution as a Taylor series. Let us define now the resolvent of the operator $H$ :

$$
R_{\lambda}(H)=(H-\lambda I)^{-1}
$$

where $\lambda$ is a complex parameter.

Now, let us consider the change of variable $\mu=\lambda^{-1}$. Then, the resolvent is written as

$$
\begin{array}{r}
R_{\lambda}(H)=(H-\lambda I)^{-1}=-\frac{1}{\lambda}\left(I-\frac{1}{\lambda} H\right)^{-1} \\
=-\mu(I-\mu H)^{-1}=\tilde{R}_{\mu}(H) .
\end{array}
$$

It is clear that the solution of equation (1) can be written in terms of the resolvent operator. Let us define $v(\mu)=-\tilde{R}_{\mu} b$. When $\mu=1$, then $\lambda=1$ and the solution of (1) is $u=v(1)$.

In general, explicitly computing the resolvent is not feasible for large electromagnetic scattering problems, since it involves inverting a huge matrix. Instead, we will find approximations based on the Taylor expansion of the resolvent. Expanding the resolvent as a Taylor series on the variable $\mu$ centered at zero we obtain [4, p. 247]

$$
\tilde{R}_{\mu}(H)=-\mu \sum_{k=0}^{\infty} \mu^{k} H^{k},
$$

assuming that the series converges. This expansion can be used to write $v(\mu)$ as

$$
v(\mu)=\mu \sum_{k=0}^{\infty}(\mu H)^{k} b=\mu \sum_{k=0}^{\infty} h_{k} \mu^{k}
$$


with $h_{k}=H^{k} b$ the vector coefficients of the Taylor series in $\mu$. If we set $\mu=1$ so that $u=v(1)$, then we recover the Neumann series (3). Thus, the Neumann series is nothing but a Taylor series evaluated at a certain point.

From the convergence analysis of this Taylor series we can recover the convergence condition for the Neumann series: a Taylor series centered at $\mu_{0}$ is convergent in an open disk where the function is holomorphic (it can be infinitely derived) [26]. Hence, the Taylor series is convergent if $\left|\mu-\mu_{0}\right|<D$, where $D$ is the radius of convergence given by the distance between $\mu_{0}$ and the closest singularity of the function. In this case, these singularities are associated with the values of $\lambda$ for which $H-\lambda I$ is singular, which correspond to $\lambda_{i}$. Recalling that $\mu_{0}=0$, that $\mu=\lambda^{-1}$, and noting $\mu_{i}=\lambda_{i}^{-1}$, we can deduce that the series is convergent and (7) holds when the following equivalent conditions are fulfilled

$$
|\mu|<\left|\mu_{i}\right|, \quad|\lambda|>\left|\lambda_{i}\right| \quad \forall i .
$$

When $\mu=1$, the condition (8) simplifies to $\left|\lambda_{i}\right|<1$ or $\left|\mu_{i}\right|>$ 1 , which is equivalent to the spectral radius condition $\rho(H)<$ 1.

Furthermore, spectral analysis also gives us information about how fast the series converges towards the solution. It can be shown [27] that, if (7) holds, the truncation error is

$$
\epsilon(\mu, K)=\left\|v(\mu)-\hat{v}_{K}(\mu)\right\|=O\left\{\left(\frac{|\mu|}{\min _{i}\left|\mu_{i}\right|}\right)^{K}\right\},
$$

where $\hat{v}_{K}(\mu)$ is defined as the truncation of (7) of order $K$

$$
\hat{v}_{K}(\mu)=\mu \sum_{k=0}^{K}(\mu H)^{k} b .
$$

The ratio $|\mu| / \min _{i}\left|\mu_{i}\right|$ is known as the asymptotic convergence factor or simply the convergence factor [18, p. 125], [28, p. 106]. Equivalently, the convergence factor can be written in terms of $\lambda$ as $\max _{i}\left|\lambda_{i}\right| /|\lambda|$, by using $\min _{i}\left|\mu_{i}\right|=$ $1 / \max _{i}\left|\lambda_{i}\right|$. Ideally, for fast convergence, we would like the convergence factor to be as small as possible. Note that the convergence factor is usually expressed in terms of the eigenvalues $\lambda_{i}$ instead of their inverses. For the case $\mu=1$, the convergence factor is equal to the spectral radius of the matrix, which is the statement usually found in literature.

\section{B. Acceleration}

In this section, we will introduce a technique for accelerating the convergence of (7). The objective is to apply a change of variable that decreases the convergence factor, so that convergence improves. Let us consider a new variable $\tilde{\mu}$ which is linked to the original variable $\mu$ by certain change of variable $\tilde{\mu}=t(\mu)$, with $t^{-1}(0)=0$. The reciprocal variable is defined as $\tilde{\lambda}=1 / \tilde{\mu}$. Let us assume that the inverse of the function $t^{-1}$ can be computed and is analytic on a certain domain that includes the value of interest for $\tilde{\mu}$. Then, we can write

$$
v(\mu)=v\left(t^{-1}(\tilde{\mu})\right)=\mu \sum_{k=0}^{\infty}\left(t^{-1}(\tilde{\mu}) H\right)^{k} b .
$$

Since the function $t^{-1}$ is locally analytic, we can compute its Taylor series centered at zero. Also, it will be possible to compute the Taylor expansion of the powers of that function. Then, the Taylor series centered at zero for the $k$-th power of $t^{-1}(\tilde{\mu})$ can be written as

$$
\left(t^{-1}(\tilde{\mu})\right)^{k}=\left.\sum_{l=0}^{\infty} \frac{1}{l !} \frac{\partial^{l}\left(t^{-1}(\tilde{\mu})\right)^{k}}{\partial \tilde{\mu}^{l}}\right|_{\tilde{\mu}=0} \tilde{\mu}^{l}
$$

We can use the Generalized Leibniz Rule [29] to write the $l$-th derivative of $\left(t^{-1}(\tilde{\mu})\right)^{k}$ as

$$
\frac{\partial^{l}\left(t^{-1}(\tilde{\mu})\right)^{k}}{\partial \tilde{\mu}^{l}}=\sum_{j_{1}+j_{2}+\cdots+j_{k}=l}\left(\begin{array}{c}
l \\
j_{1}, \ldots, j_{k}
\end{array}\right) \prod_{i=1}^{k} \frac{\partial^{j_{i}} t^{-1}(\tilde{\mu})}{\partial \tilde{\mu}^{j_{i}}}
$$

where the summation runs over the $k$-tuples of non-negative integers that fulfill $\sum j_{i}=l$. We will distinguish two different cases for (13) when evaluated at $\tilde{\mu}=0: l<k$ and $l \geq k$. If $l<k$, since $j_{i}$ are non-negative integers, then at least one of the indices $j_{i}$ should be zero to fulfill $\sum j_{i}=l$, for all the $k$-tuples. This means that the zero order derivative will appear at least once on the product of (13). Then, taking into account that $t^{-1}(0)=0$, the $l$-th derivative of $\left(t^{-1}(\tilde{\mu})\right)^{k}$ evaluated at $\tilde{\mu}=0$ will be zero if $l<k$, since at least one of the terms of the product will be zero. However, in the case $l \geq k$ this does not apply, and the derivative will not be zero in general.

By applying the reasoning above, the terms of series (12) where $l<k$ can be neglected, as they are zero. Thus, we can write (12) as

$$
\begin{aligned}
& \left(t^{-1}(\tilde{\mu})\right)^{k}=\left.\sum_{l=k}^{\infty} \frac{1}{l !} \frac{\partial^{l}\left(t^{-1}(\tilde{\mu})\right)^{k}}{\partial \tilde{\mu}^{l}}\right|_{\tilde{\mu}=0} \tilde{\mu}^{l} \\
& =\left.\tilde{\mu}^{k} \sum_{j=0}^{\infty} \frac{1}{(k+j) !} \frac{\partial^{k+j}\left(t^{-1}(\tilde{\mu})\right)^{k}}{\partial \tilde{\mu}^{k+j}}\right|_{\tilde{\mu}=0} \tilde{\mu}^{j}=\tilde{\mu}^{k} \sum_{j=0}^{\infty} \beta_{j}^{k} \tilde{\mu}^{j},
\end{aligned}
$$

where we have introduced the coefficients $\beta_{j}^{k}$ for compactness (note that $k$ in $\beta_{j}^{k}$ is a superindex, not an exponent as in $\tilde{\mu}^{k}$ ).

Now, we can insert (14) into (11) to obtain

$$
\begin{aligned}
v\left(t^{-1}(\tilde{\mu})\right) & =\mu \sum_{k=0}^{\infty} \tilde{\mu}^{k} \sum_{j=0}^{\infty} \beta_{j}^{k} \tilde{\mu}^{j} H^{k} b= \\
& =\mu \sum_{n=0}^{\infty}\left(\sum_{k=0}^{n} \beta_{n-k}^{k}\left(H^{k} b\right)\right) \tilde{\mu}^{n}=\mu \sum_{n=0}^{\infty} a_{n} \tilde{\mu}^{n}
\end{aligned}
$$

where the vector coefficients $a_{n}$ of the Taylor series in $\tilde{\mu}$

$$
a_{n}=\sum_{k=0}^{n} \beta_{n-k}^{k} h_{k}
$$

are linear combinations of the coefficients of the Taylor series in $\mu$ (7). In (15) we have reordered an expansion in terms of $\mu$ to obtain an expansion in terms of $\tilde{\mu}$. According to the theorem of composition of formal power series [26], the $a_{n}$ coefficients of the series (15) in $\tilde{\mu}$ are exactly the same as those resulting from direct derivation of the Taylor series expansion of $v\left(t^{-1}(\tilde{\mu})\right)$.

Note that these $a_{n}$ coefficients can be computed exactly for a finite $n$, since each of them is written as a finite series of $n$ terms. This is due to the presence of the common factor $\tilde{\mu}^{k}$ 
in the final expression of (14), which in turn is a consequence of $t^{-1}(0)=0$, as we have explained before.

The convergence conditions of (15) should now be studied in terms of of the new variable $\tilde{\mu}$. Both the singularities and the point $\mu$ are relocated by the mapping $t$, that represents the change of variable. Thus, the singularities $\mu_{i}=1 / \lambda_{i}$ associated to the eigenvalues of $H$ correspond now to the points $\tilde{\mu}_{i}=t\left(\mu_{i}\right)$. The new convergence condition is now $|\tilde{\mu}|<\left|\tilde{\mu}_{i}\right|$. Similarly, the new convergence factor is $|\tilde{\mu}| / \min _{i}\left|\tilde{\mu}_{i}\right|$. In terms of $\tilde{\lambda}$, defining $\tilde{\lambda}_{i}=1 / \tilde{\mu}_{i}$ and recalling that $\tilde{\lambda}=1 / \tilde{\mu}$, the convergence condition is $|\tilde{\lambda}|>\left|\tilde{\lambda}_{i}\right|$ and the convergence factor $\max _{i}\left|\tilde{\lambda}_{i}\right| /|\tilde{\lambda}|$.

The truncation error is

$\epsilon(\tilde{\mu}, K)=\left\|v\left(t^{-1}(\tilde{\mu})\right)-\hat{v}_{K}\left(t^{-1}(\tilde{\mu})\right)\right\|=O\left\{\left(\frac{|\tilde{\mu}|}{\min _{i}\left|\tilde{\mu}_{i}\right|}\right)^{K}\right\}$,

where $\hat{v}_{K}\left(t^{-1}(\tilde{\mu})\right)$ is the truncation of the infinite summation in (15) at $n=K$, similarly to (10).

In this technique, the objective is to find a change of variable $t$ such that the error (17) decreases faster than the error (9) for the point of interest $\tilde{\mu}=t(1)$, since $v(\mu=1)$ is the solution of (1) [30].

Ideally, we would like the change of variable $t$ to minimize the convergence factor associated to $\tilde{\mu}$. Selecting a suitable change of variable is not easy since, in general, it requires a knowledge of the position of the singularities in the complex plane, which implies knowing the spectrum of $H$. The exact computation of the eigenvalues of a general matrix $H$ is unfeasible due to its computational cost for the large matrices under interest here. However, in the case of operators arising from physical problems we can sometimes find analytical ways to locate the spectrum in a certain region of the complex plane. For example, many analytic results about the spectrum of continuous and discretized electromagnetic integral operators may be found in [31].

It is possible to let the change of variable $t$ depend on one or more parameters. The advantage of parametric changes of variable is that they can be heuristically adjusted to improve convergence. As an example, we propose three parametric changes of variable, similar to the ones that may be found in literature [32], [33]

1) Change 1: We define the change of variable $t_{1}$ depending on a parameter $y$ as

$$
\tilde{\mu}=t_{1}(\mu)=\frac{\mu}{\mu+y} ; \quad \mu=t_{1}^{-1}(\tilde{\mu})=y \frac{\tilde{\mu}}{1-\tilde{\mu}} .
$$

By using the Maclaurin expansion of $(1-x)^{-m}$ [34] we can find the coefficients $\beta_{j}^{k}$ of series (15)

$$
\beta_{j}^{k}=y^{k}\left(\begin{array}{c}
k+j-1 \\
j
\end{array}\right)
$$

With this, it is trivial to compute the coefficients $a_{n}$.

2) Change 2: In this case the change of variable is

$$
\begin{aligned}
& \tilde{\mu}=t_{2}(\mu)=\frac{y+2 \mu-\sqrt{y^{2}+4 y \mu}}{2 \mu} \\
& \mu=t_{2}^{-1}(\tilde{\mu})=y \frac{\tilde{\mu}}{(1-\tilde{\mu})^{2}}
\end{aligned}
$$

Note that, in this case, the change of variable $t_{2}$ is easily seen to be a solution of (21). The fact that we choose the minus sign in the square root ensures that we are in the region where the Maclaurin series for (21) is convergent. The $\beta_{j}^{k}$ coefficients for this case are very similar to the previous one, since $t_{1}^{-1}$ and $t_{2}^{-1}$ only differ by an exponent in the denominator. In this case, we will have

$$
\beta_{j}^{k}=y^{k}\left(\begin{array}{c}
2 k+j-1 \\
j
\end{array}\right) .
$$

3) Change 3: This third change will be defined as

$$
\tilde{\mu}=t_{3}(\mu)=\frac{\sqrt{\mu+y^{2}}-y}{\sqrt{\mu+y^{2}}+y} ; \quad \mu=t_{3}^{-1}(\tilde{\mu})=y^{2} \frac{4 \tilde{\mu}}{(1-\tilde{\mu})^{2}} .
$$

In this case, the coefficients for (15) can be written as

$$
\beta_{j}^{k}=4^{k} y^{2 k}\left(\begin{array}{c}
2 k+j-1 \\
j
\end{array}\right) .
$$

With this change of variable, if $y$ is real, then $\mu_{i}$ are real and negative and smaller than $-y^{2}$, so all the singularities are mapped outside the unit circle.

\section{Rearranged series}

Up to this point, we have obtained a power series expansion of the resolvent (II-A), which allows to compute the solution of the system (1) when evaluated at $\mu=1$, provided that the series converges. Then, we have applied a change of variable to enhance the convergence of this method, obtaining a Taylor expansion in $\tilde{\mu}$ of coefficients $a_{n}$ (15).

The problem (1) could be directly addressed with this formulation. Nonetheless, we notice that the computation of each one of the $a_{n}$ vector coefficients in (15) involves a linear combination of the $n+1$ vectors $h_{k}=H^{k} b$. The computational cost can be reduced by rearranging the series in such a way that the $n$-th term only contains the vector $h_{n}$, multiplied by a scalar coefficient. Also, in this way we preserve the original iterative scheme based on successive orders of scattering.

We will perform this rearrangement on $\hat{v}_{K}\left(t^{-1}(\tilde{\mu})\right)$, which represents the truncation of order $K$ of the series in (15):

$$
\begin{aligned}
\hat{v}_{K}\left(t^{-1}(\tilde{\mu})\right) & =\mu \sum_{n=0}^{K} a_{n} \tilde{\mu}^{n}=\mu \sum_{n=0}^{K}\left(\sum_{k=0}^{n} \beta_{n-k}^{k} h_{k}\right) \tilde{\mu}^{n} \\
& =\mu \sum_{k=0}^{K}\left(\sum_{n=k}^{K} \beta_{n-k}^{k} \tilde{\mu}^{n}\right) h_{k}=\mu \sum_{k=0}^{K} \gamma_{k} h_{k}
\end{aligned}
$$

where the coefficients $\gamma_{k}$ represent the summation in brackets. The main difference between (15) and (25) is that the $a_{n}$ coefficients in (15) are vectors and involve several elements $h_{k}$, while coefficients $\gamma_{k}$ are scalar.

The number of operations to compute the set of coefficients $\gamma_{k}$ for $k=0 \ldots K$ is $O\left(K^{2}\right)$, where $K$ is the order of the expansion. Note that it is independent of the size of the linear system matrix, unlike in other iterative methods. 
To sum up, we gather here all the necessary formulas to compute the series coefficients:

$$
\begin{aligned}
& \hat{v}_{K}(\mu, y)=\mu \sum_{k=0}^{K} \gamma_{k} H^{k} b \\
& \gamma_{k}=\sum_{n=k}^{K} \beta_{n-k}^{k}(t(\mu))^{n} \\
& \beta_{j}^{k}=\left.\frac{1}{(k+j) !} \frac{\partial^{(k+j)}\left(t^{-1}(\tilde{\mu})\right)^{k}}{\partial \tilde{\mu}^{k+j}}\right|_{\tilde{\mu}=0}
\end{aligned}
$$

Note that we have explicitly indicated the dependence of $\hat{v}_{K}$ on $y$, the change of variable parameter. Then, the solution of the system (1), assuming convergence, is for $\mu=1$

$$
u \approx \sum_{k=0}^{K} \gamma_{k} H^{k} b
$$

which can easily be implemented as an iterative method.

At this point, it is important to highlight certain features of this series. The expression (29) fulfills the conditions we were originally looking for. It resembles a classical Neumann series except the coefficients $\gamma_{k}$, which embed the effect of the change of variable. This approach can be understood as a Krylov subspace method, since the solution is computed as a linear combination of matrix powers applied to the right hand side of the linear system. The distinct feature of our approach relies on the way of computing the coefficients: in many Krylov subspace methods, the computation of each coefficient requires the result of the previous iteration and involves matrix-vector operations, whereas here computing the coefficients $\gamma_{k}$ only requires scalar operations and does not depend on information from previous iterations. Indeed, the whole sequence $\gamma_{0}, \ldots, \gamma_{K}$ can be computed beforehand by using (27)-(28). Only the function $t$ defining the change of variable is needed. The matrix $H$ and the vector $b$ are not required in this computation.

Regarding convergence, it is worth to remark that (26) is only a rearrangement of (15). Hence, it enjoys the same convergence properties. The series (29) monotonically converges with $K$, if the convergence conditions for $\tilde{\mu}$ are fulfilled.

\section{Application to Electromagnetic Case}

The Born series, as well as other methods based on iterative scattering, implicitly relies on a Neumann series of the form (3), which usually is implemented in an iterative way, similarly to (2). In this section, we will apply this technique to electromagnetic problems and, after a change of variables, will obtain a modified Born series with accelerated convergence.

First, we will consider the scattering produced by a set of disjoint objects by using the Generalized Born Series [6], [7]. The scattering operator is discretized by the Method of Moments (MoM) [35] and the global problem is solved by a summation of successive orders of scattering. One of the most interesting features of this formulation is that it can be applied as a domain decomposition method [8].
For the sake of simplicity, let us assume a PEC material. MoM discretization of the Electric Field Integral Equation (EFIE) [36] leads to the linear system

$$
Z^{(E)} J=-E_{i},
$$

where $Z$ is the impedance matrix, $E_{i}$ is the incident field vector and $J$ is the unknown vector of induced currents.

We can split the linear system matrix $Z^{(E)}$ into submatrices $\mathbf{Z}_{i j}$, where $\mathbf{Z}_{i i}$ represent the self-interaction matrix of object $i$ and $\mathbf{Z}_{i j}$ the interaction between objects $i$ and $j$. Then, the solution $J$ can be written as a Born series:

$$
J=Z_{d} \sum_{k=0}^{\infty}\left(Z_{s} Z_{d}\right)^{k} E_{i}
$$

where the auxiliary matrices $Z_{d}$ and $Z_{s}$ are

$$
\begin{gathered}
Z_{d}=\left[\begin{array}{cccc}
-\mathbf{Z}_{11}^{-1} & \mathbf{0} & \ldots & \mathbf{0} \\
\mathbf{0} & -\mathbf{Z}_{22}^{-1} & \ldots & \mathbf{0} \\
\vdots & & \ddots & \\
\mathbf{0} & \ldots & & -\mathbf{Z}_{n n}^{-1}
\end{array}\right] \\
Z_{s}=\left[\begin{array}{cccc}
\mathbf{0} & \mathbf{Z}_{12} & \ldots & \mathbf{Z}_{1 n} \\
\mathbf{Z}_{21} & \mathbf{0} & \ldots & \mathbf{Z}_{2 n} \\
\vdots & & \ddots & \vdots \\
\mathbf{Z}_{n 1} & \ldots & & \mathbf{0}
\end{array}\right]
\end{gathered}
$$

Comparing with eq. (3), in this case the iteration matrix is $H=Z_{s} Z_{d}$. By applying the matrix $Z_{s} Z_{d}$ to the incident field vector we obtain the field scattered by the set of objects individually considered, disregarding interactions between them. This first order scattered field becomes the incident field of the second order interactions at the next iteration and, therefore, the terms of the Born series (31) correspond to the successive orders of scattering approach [6], [7].

Besides, we can identify this method with a blockpreconditioned Jacobi [28]. The matrix $Z_{d}$, which naturally arises from the Born formulation, acts as a preconditioner.

The Born series (31) converges when the spectral condition is met, that is, when $\rho\left(Z_{s} Z_{d}\right)<1$. From a physical point of view, this means that the interaction between different objects is weak.

By employing the acceleration process described in the previous section, we will be able to extend the range of application for this Generalized Born series: the term $\left(Z_{s} Z_{d}\right)^{k}$ will be substituted by $\gamma_{k}\left(Z_{s} Z_{d}\right)^{k}$, where $\gamma_{k}$ is computed with the expressions (27)-(28). Finally, the accelerated series will be

$$
J=Z_{d} \sum_{k=0}^{\infty} \gamma_{k}\left(Z_{s} Z_{d}\right)^{k} E_{i}
$$

The Magnetic Field Integral Equation (MFIE) is a 2nd kind Fredholm IE [31], so it naturally decomposes into an equation of the form (1), that in functional form is

$$
J=2 \mathcal{K}(J)+2 \hat{n} \times H_{i}
$$

where $\mathcal{K}$ is the operator computing the principal value of the scattered field. The operator $2 \mathcal{K}$, or its corresponding MoM 
discretization, plays the role of the iteration matrix $H$. The expression for the accelerated series is

$$
J=\sum_{k=0}^{\infty} \gamma_{k} 2 \mathcal{K}\left(2 \hat{n} \times H_{i}\right)
$$

It is also possible to apply this technique to 1 st kind Fredholm integral equations, like the EFIE, by directly setting the iteration matrix $H$ to $H=I-Z$, where $Z$ is the linear system matrix, as in the electrostatic problem example that follows below.

\section{NUMERICAL EXAMPLES}

\section{A. Example 1}

The objective of the first example is to compare different changes of variable. In Section II-B, we proposed three mappings named $t_{1}, t_{2}$, and $t_{3}$. We will denote the associated changes of variable as $C V 1, C V 2$, and $C V 3$. These three changes of variable will be applied to accelerate the Born series for two different physical problems.

1) Static case: The first case corresponds to the electrostatic problem of a perfectly conducting straight wire of circular cross section set at potential $V_{0}=1 \mathrm{~V}$. The wire length and radius are respectively $L=10 \mathrm{~m}$ and $a=0.001 \mathrm{~m}$.

Since $a \ll L$ we use the thin wire approximation as in [36] and force to $V_{0}$ the potential at the cylinder axis created by the cylindrical surface charge

$$
V(z)=\frac{1}{4 \pi \varepsilon_{0}} \int_{0}^{2 \pi} \int_{-L / 2}^{L / 2} \frac{\sigma\left(z^{\prime}\right)}{\sqrt{\left(z-z^{\prime}\right)^{2}+a^{2}}} d z^{\prime} d \phi^{\prime}=V_{0}
$$

Using MoM, the unknown $\sigma\left(z^{\prime}\right)$ is expanded into 100 equal rectangular pulse subdomain basis functions of length $L / 100$ and the integral equation (37) is discretized into a linear system $Z \sigma=V_{0}$. This system can be converted into an expression of the form (1) by defining an iteration matrix $H=I-Z$, so that $\sigma=H \sigma+V_{0}$. It is expected that the conventional Born series (3) will not converge in this case. We will apply instead the modified series (29) proposed in this work.

2) Dynamic case: The second case is a $2 \mathrm{D}$ problem consisting an array of $4 \times 4$ infinite circular cylinders forming a square lattice. The incident field is a plane wave propagating in a direction perpendicular to the cylinders axis and parallel to the rows of the array. The electric field polarization is parallel to the cylinders axis (TM). The radius of all the cylinders is $0.305 \lambda$, where $\lambda$ is the wavelength. The distance between the center of any cylinder and the center of the closest neighbors is $3.655 \lambda$.

The 2D EFIE-TM [36] is discretized by MoM to obtain a linear system of the form (30). The boundary of each cylinder is discretized into 100 equal segments corresponding to rectangular pulse basis functions. In this case, we will solve the linear system using a generalized Born series of the form (31), where the matrix blocks correspond to the individual cylinders.

All three changes of variable are applied to both the static and dynamic problems, and the results are shown in Fig. 1. The value of the parameter $y$ in these examples has been optimized
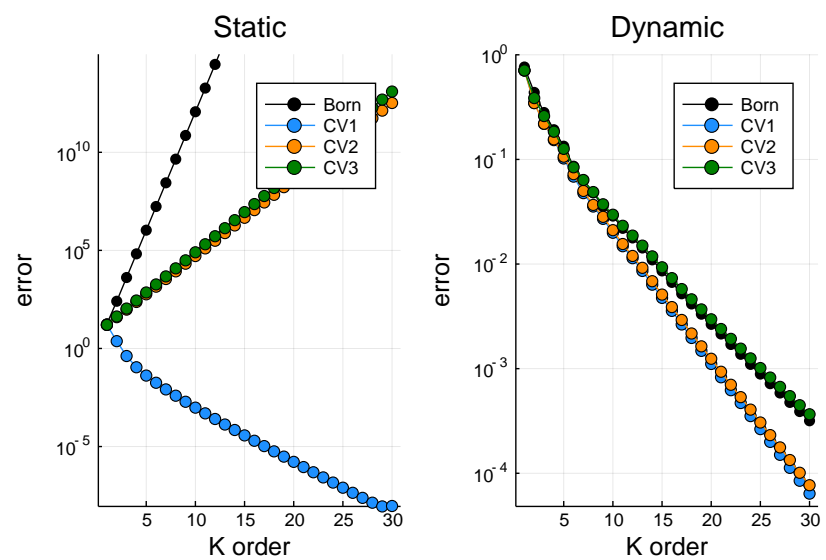

Fig. 1. Convergence comparison for three different changes of variable applied to the static and dynamic cases of example 1 . Relative error versus iteration number for the changes $C V 1, C V 2$ and $C V 3$ compared with the conventional Born series.

to achieve the fastest convergence. The convergence behavior as a function of $y$ in the complex plane is addressed for the examples in Section III-B and Section III-C.

TABLE I

PARAMETER VALUES FOR THE CHANGES OF VARIABLE IN FIG. 1

\begin{tabular}{|c|c|c|} 
& Static & Dynamic \\
\hline CV1 & 0.05 & $3.44-2.62 i$ \\
CV2 & $0.23+0.22 i$ & $5.13-4.42 i$ \\
CV3 & $0.28+0.10 i$ & $5.10+1.35 i$ \\
\hline
\end{tabular}

TABLE II

\begin{tabular}{|c|c|c|c|}
\hline & Ordered & Disordered & Ellipse \\
\hline$\gamma_{0}$ & $1.0 \measuredangle 0^{\circ}$ & $1.0 \measuredangle 0.0^{\circ}$ & $1.0 \measuredangle 0.0^{\circ}$ \\
\hline$\gamma_{1}$ & $1.0005 \measuredangle-0.00063^{\circ}$ & $1.0 \measuredangle 0.0001^{\circ}$ & $1.0 \measuredangle 0.0428^{\circ}$ \\
\hline$\gamma_{2}$ & $1.0063 \measuredangle \quad 0.1330^{\circ}$ & $1.0 \measuredangle 0.0003^{\circ}$ & $1.0140 \measuredangle-0.0344^{\circ}$ \\
\hline$\gamma_{3}$ & $1.0257 \measuredangle$ & $1.0 \measuredangle 0.0069^{\circ}$ & $0.9959 \measuredangle-6.7682^{\circ}$ \\
\hline$\gamma_{4}$ & $1.0264 \measuredangle$ & $0.9998 \measuredangle 0.0812^{\circ}$ & $0.4390 \measuredangle \quad 9.3805^{\circ}$ \\
\hline$\gamma_{5}$ & $0.9116 \measuredangle$ & $0.9955 \measuredangle 0.0 .5660^{\circ}$ & $2.1366 \measuredangle \quad 54.8786^{\circ}$ \\
\hline$\gamma_{6}$ & $0.6388 \measuredangle$ & $0.9637 \measuredangle 2.4877^{\circ}$ & $4.5547 \measuredangle-4.1135^{\circ}$ \\
\hline$\gamma_{7}$ & $0.3173 \measuredangle$ & $0.8383 \measuredangle 7.3402^{\circ}$ & $4.7450 \measuredangle-76.4610^{\circ}$ \\
\hline$\gamma_{8}$ & $0.0977 \measuredangle$ & $0.5531 \measuredangle 15.7890^{\circ}$ & $2.6125 \measuredangle-155.4010^{\circ}$ \\
\hline$\gamma_{9}$ & $131.207^{\circ}$ & $0.1949 \measuredangle 27.6386^{\circ}$ & $0.6114 \measuredangle \quad 121.4020^{\circ}$ \\
\hline
\end{tabular}

VALUES FOR THE $\gamma_{k}$ COEFFICIENTS FOR $K=9$.

In the static case, the Born series rapidly diverges. The only change of variable capable of achieving convergence is $C V 1$. $C V 2$ and $C V 3$ reduce the convergence factor with respect to the Born series, but not to the point of achieving convergence.

In the dynamic case, the Born series converges. $C V 1$ and $C V 2$ outperform the Born series by almost an order of magnitude, while $C V 3$ performs very similar to the Born series itself.

These two cases (static and dynamic) correspond to two completely different physical problems, and thus the spectral properties of their impedance matrices is completely different (in fact, the eigenvalues for the electrostatic case are real, as the matrix is hermitian [37]). Depending on the characteristics of the system, it is more convenient to choose one or another change of variable. 
B. Example 2

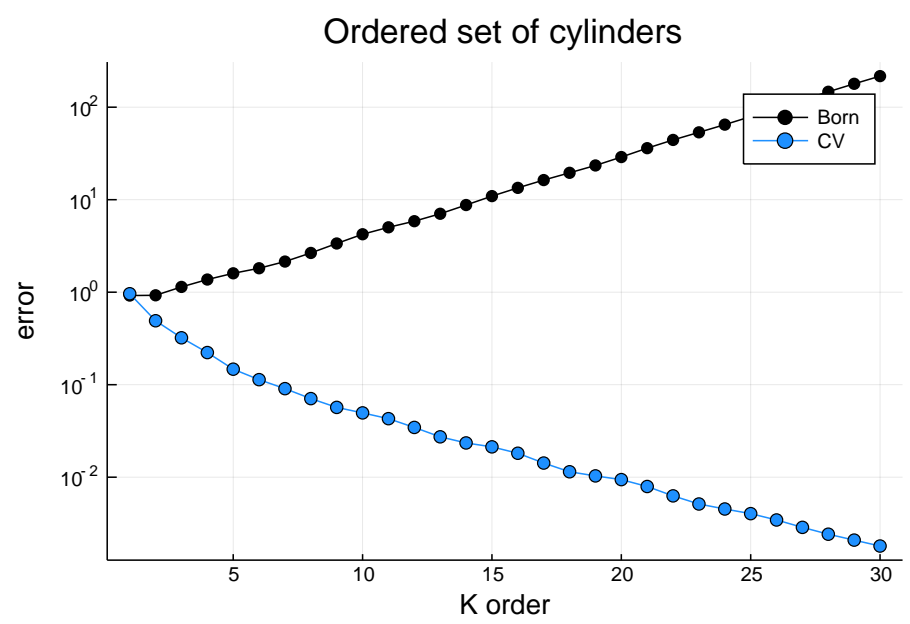

Fig. 2. Relative error versus expansion order (or number of iterations) for the ordered set of cylinders in example 2. The conventional Born series diverges, whereas the modified series with change of variable $C V 1$ and parameter $y=1.18+0.85 i$ converges.
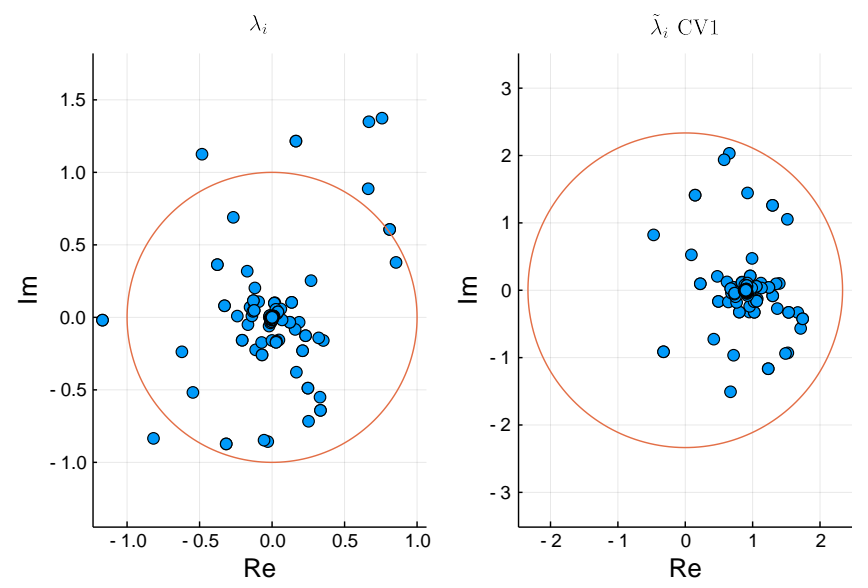

Fig. 3. Blue points represent the singularities $\lambda_{i}$ given by the eigenvalues of the iteration matrix $H=Z_{s} Z_{d}$ (left). The radius of the red circumference corresponds to $|\lambda|$ for the case of interest $\mu=1 / \lambda=1$. The condition $|\lambda|>\left|\lambda_{i}\right|$ is not met (some $\lambda_{i}$ lie outside the circle), and thus the series does not converge. After the change of variable (right) the convergence condition $|\tilde{\lambda}|>\left|\tilde{\lambda}_{i}\right|$ is fulfilled (the values $\tilde{\lambda}_{i}$ lie within the circle of radius $|\tilde{\lambda}|=2.3$ ). The convergence factors for the two cases are $\max _{i}\left|\lambda_{i}\right| /|\lambda|=1.57>1$ and $\max _{i}\left|\tilde{\lambda}_{i}\right| /|\tilde{\lambda}|=0.91<1$ for the original and modified series, respectively.

In this second example we will study the effect of the change of variable from the spectral point of view. We will consider two sets of circular cylinders. The first set, referred to as "ordered array", is very similar to the dynamic case in Section III-A. It also consists of a $4 \times 4$ square array of identical cylinders, but in this case the radius is $0.205 \lambda$, and the distance between the centers of neighbor cylinders is 1.191 $\lambda$. Each cylinder is discretized into 100 equal segments and the 2D EFIE-TM is discretized by MoM.

The second case, "disordered array" consists of 25 cylinders of the same radius as the ones from the ordered array, but in this case they are randomly arranged in a disordered way (see the small white circles in Fig. 5). In both the ordered and
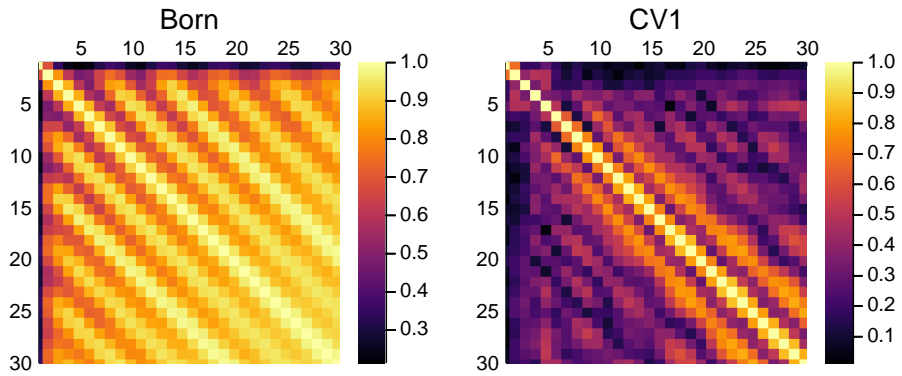

Fig. 4. Absolute value of Gram matrix elements for the normalized implicit basis formed by the vectors $h_{k} /\left\|h_{k}\right\|$ generated by the original Born series (left) and by the vectors $a_{k} /\left\|a_{k}\right\|$ generated by the modified series using $C V 1$ (right), for the ordered array of example 2. The Neumann expansion (7) does not generate a basis of linearly independent vectors, whereas (15) does.

disordered array, the incident field is the same plane wave as in the dynamic case of Section III-A.

For the ordered set, we can see in Fig. 2 that the conventional Born series is divergent, whereas the series modified with the change of variable CV1 with parameter $y=1.18+$ $0.85 i$ converges. The effect of this transform on the spectrum of the iteration matrix is shown in Fig. 3: the change of variable maps the singularity into the circle of radius $t_{1}(1)$, which entails convergence.

It is worth to pay attention to the orthogonality relations between the terms of both the original and the modified Born series. We can understand the vectors generated by the iterative method as the implicit basis upon which we write our solution. In the case of the original Born series (7), this basis is formed by the vectors $h_{k}$, which are recognized as the standard Krylov subspace basis. In the modified Born series (15), the basis is formed by the vectors $a_{n}$.

Fig. 4 shows the Gram matrix of the normalized vectors corresponding to the first 30 terms in the Born series (7) and the modified series (15). The original Born series generates a set of vectors that, from a numerical point of view, are linearly dependent (the pseudo-rank of the matrix formed by the 30 vectors of the implicit basis $h_{k}$ is 25). Thus, the plain Born series does not generate a good basis for the solution, and the iteration diverges. However, after applying the change of variable $C V 1$, the iterative method generates linearly independent vectors, which constitute a good basis for the solution. Thus, although our method is not intended to find an orthogonal basis, it reduces the linear dependence between the implicit basis vectors. In this way, the change of variable plays a similar role to orthogonalization procedures in Krylov subspace iterative methods.

In the second case, we study the disordered set of cylinders. Fig. 5 shows the cylinders arrangement and the total field (incident plus scattered). The convergence results for change of variable $C V 1$ and parameter $y=4.60+1.50 i$ in Fig. 6 are similar to the ones for the ordered set, with the difference that here the Born series is already convergent, and the change of variable only accelerates convergence. The improvement of the convergence factor from 0.90 to 0.65 is shown in the operator spectrum of Fig. 7. The better orthogonality between the iteration implicit basis vectors is visible in the Gramm 


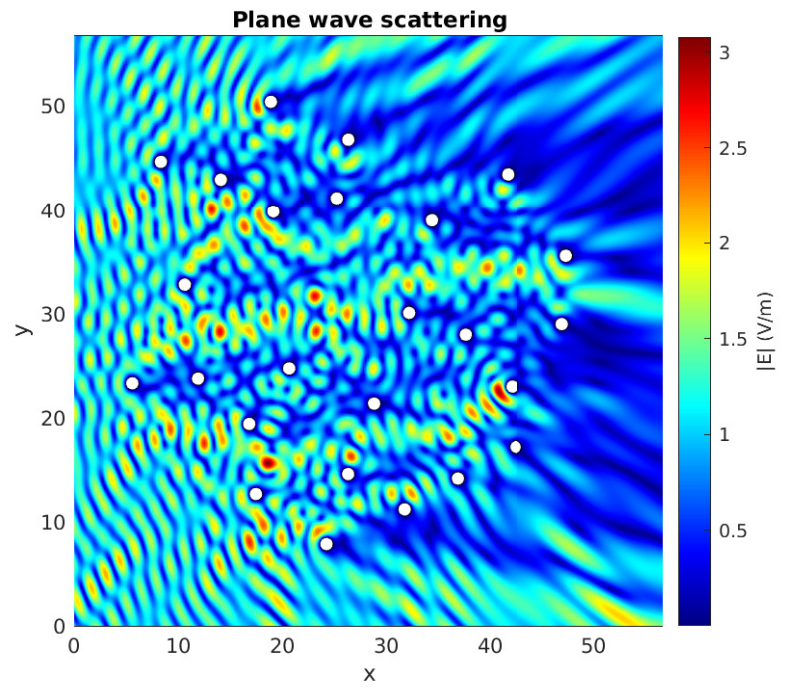

Fig. 5. Total field for the disordered set of cylinders of example 2, illuminated by a plane wave propagating in the $x$ direction. The cylinders are represented as small white circles.

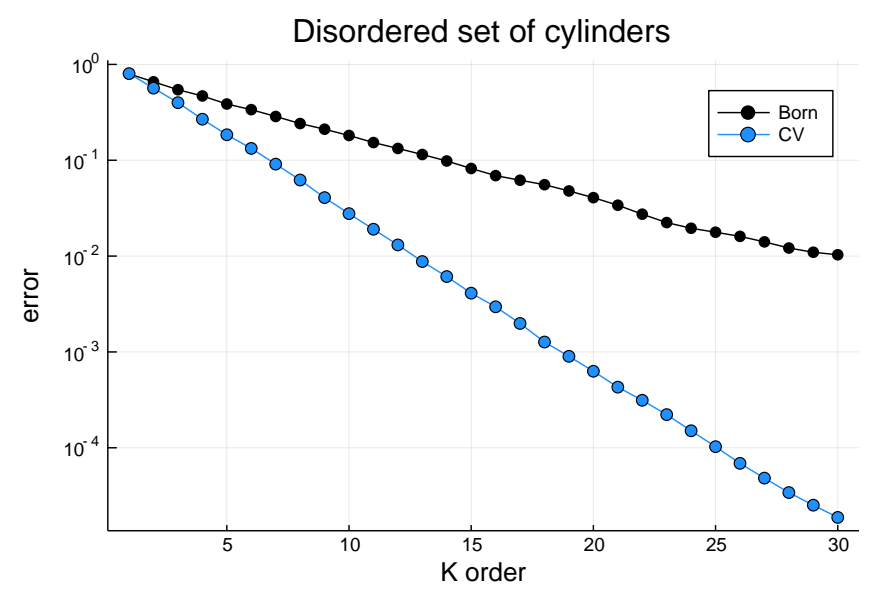

Fig. 6. Relative error versus expansion order (or number of iterations) for the disordered set of cylinders in example 2. Both the conventional Born series and the modified series with change of variable $C V 1$ and parameter $y=$ $4.60+1.50 i$ converge, but the change of variable accelerates convergence.

matrices shown in Fig. 8.

Table II shows the values of the $\gamma_{k}$ coefficients for $K=$ 9 , both for the ordered set and for the disordered set. These coefficients are employed in (34).

The change of variable parameter $y$ in these examples has been optimized to achieve the fastest convergence. Fig. 10 and 11 show the relative error after 30 iterations as a function of the $y$ parameter for the ordered and disordered arrays, respectively. The convergence is well behaved and regions with fast convergence are relatively wide, which allows to easily find good values for the parameter.

It is also worth to note that the parameter depends essentially on the operator spectrum, so it must be computed only once in problems involving multiple incident fields, like monostatic RCS. Techniques for efficiently tuning the parameter or parameters of a change of variable will be described in a forthcoming publication.
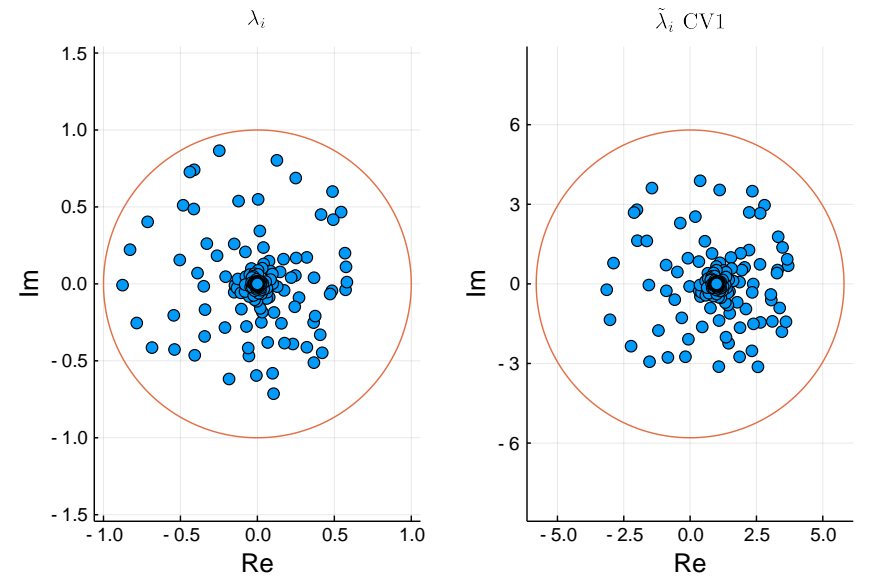

Fig. 7. Singularities $\lambda_{i}$ given by the eigenvalues of the iteration matrix $H=Z_{s} Z_{d}$ for the disordered array of Example 2 (left) and transformed singularities $\tilde{\lambda}_{i}$ (right). The convergence factor improves from 0.90 in the original Born series (left) to 0.65 in the modified one (right).
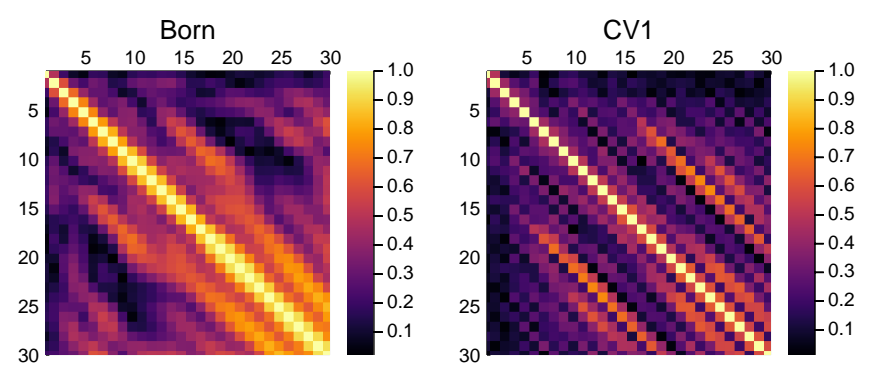

Fig. 8. Absolute value of Gram matrix elements for the normalized implicit basis formed by the vectors $h_{k} /\left\|h_{k}\right\|$ generated by the original Born series (left) and by the vectors $a_{k} /\left\|a_{k}\right\|$ generated by the modified series using $C V 1$ (right), for the disordered array of example 2. The change of variable improves the orthogonality of the iterative procedure implicit basis.

\section{Example 3}

In this case, we will apply the change of variable CV1 to solve the MFIE for a 2D perfectly conducting ellipse under TM polarization. The major and minor semi-axis have length $0.22 \lambda$ and $0.13 \lambda$, respectively. The incident field is a TM plane wave with incidence direction parallel to the minor semi-axis. The MFIE is discretized by MoM using 501 points.

As we can see in Fig. 9, the Born series based on the second kind Fredholm integral equation for (35) diverges. However, applying the accelerated series (36) with $C V 1(y=-0.24-$ $2.09 i)$ leads to convergence. Fig. 12, shows the convergence as a function of the complex parameter $y$.

In some cases, a simple change of variable as CV1, CV2 or $\mathrm{CV} 3$ in this work may not be enough to achieve convergence, due to the complexity of the spectrum. In those cases, more complex changes of variable are necessary. Changes based on Schwarz-Christoffel transformations with several parameters show a good compromise between performance and computational cost [38]. We have added the relative error of a Schwarz-Christoffel change of variable (SC) to Fig. 9 in order to illustrate the improved performance of more sophisticated techniques. 


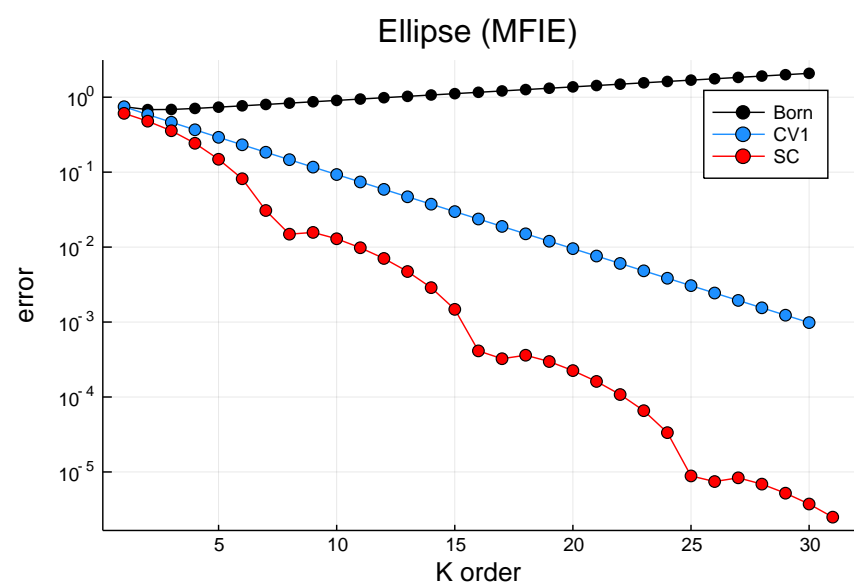

Fig. 9. Relative error versus expansion order (or number of iterations) for the ellipse under MFIE formulation of Example 3. Born series diverges and $C V 1$ with $y=-0.24-2.09 i$ converges. The convergence for a change of variable based on a Schwarz-Christoffel transformation of 9 parameters (similarly as in [38]) is also shown (SC).

\section{Rel. error after 30 iterations (ordered set)}
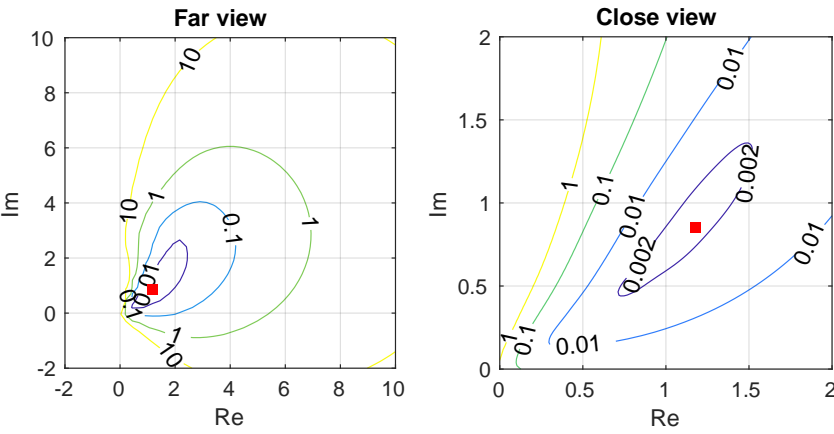

Fig. 10. Isolines showing the relative error after 30 iterations for the ordered array (Example 2) as a function of the complex parameter $y$. The red square shows the optimum value $y=1.18+0.85 i$.

Rel. error after 30 iterations (disordered set)
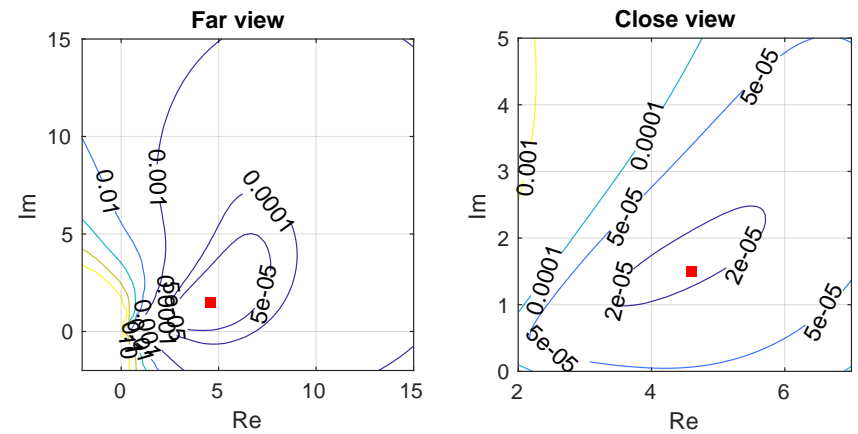

Fig. 11. Isolines showing the relative error after 30 iterations for the disordered array (Example 2) as a function of the complex parameter $y$. The red square shows the optimum value $y=4.60+1.50 i$.

\section{CONCLUSION}

In this paper we have proposed a technique to improve the convergence of fixed-point iteration methods -like the Born series- that are often applied to the computation of multiple scattering, as some Domain Decomposition methods [6], [8],
Rel. error after 30 iterations (ellipse)
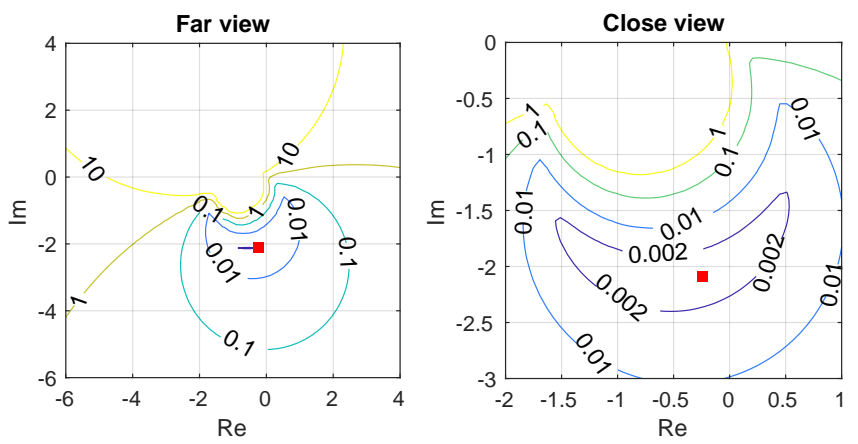

Fig. 12. Isolines showing the relative error after 30 iterations for the ellipse under MFIE formulation (Example 3) as a function of the complex parameter $y$. The red square shows the optimum value $y=-0.24-2.09 i$.

Iterative Physical Optics [13], [15], or Radiative Transfer [10]. The modified Born series proposed here improves the convergence of these methods, or even achieves convergence in cases where they would otherwise diverge, while preserving their structure and advantages.

A change of variable, based on a rough estimation of the resolvent spectrum, is applied to improve the convergence factor of the resolvent power expansion. Then, the series is rearranged to preserve the original scheme based on successive orders of scattering. Finally, all the mathematical apparatus is embedded in a set of scalar coefficients that multiply the terms of the Born series. These coefficients can be computed by applying simple recurrence relations with a minimal computational cost independent of the linear system size. This approach can be used to improve the convergence of any method based on a fixed-point iterative scheme without the need to restructure the code. Besides, the coefficients depend essentially on the operator spectrum, so they must be computed only once in problems involving multiple incident fields.

Numerical examples show that this technique, applied with very simple changes of variable, is capable of substantially improving the convergence of Born series not only for sets of objects involving significant multiple scattering, but also for the solution of integral equations with a single object, both in electrostatic and electrodynamic problems. We have also shown empirically that the acceleration procedure improves the orthogonality of the implicit basis associated to the iterative method.

\section{REFERENCES}

[1] N. Zettili, Quantum mechanics: concepts and applications, 2nd ed. Chichester, U.K: Wiley, 2009, oCLC: ocn255894625.

[2] C. S. Kubrusly, Spectral Theory of Operators on Hilbert Spaces. Boston: Birkhäuser Boston, 2012. [Online]. Available: http://link.springer.com/10.1007/978-0-8176-8328-3

[3] V. Hutson and J. S. Pym, Applications of functional analysis and operator theory, ser. Mathematics in science and engineering. London ; New York: Academic Press, 1980, no. no. 146.

[4] M. Renardy and R. C. Rogers, An introduction to partial differential equations, 2nd ed., ser. Texts in applied mathematics. New York: Springer, 2004, no. 13.

[5] G. J. Gbur, Mathematical Methods for Optical Physics and Engineering. Cambridge University Press, 2011. 
[6] G. T. Schuster, "A hybrid BIE+Born series modeling scheme: Generalized Born series," The Journal of the Acoustical Society of America, vol. 77, no. 3, pp. 865-879, Mar. 1985. [Online]. Available: http://asa.scitation.org/doi/10.1121/1.392055

[7] P. A. Martin, Multiple scattering: interaction of time-harmonic waves with $N$ obstacles, ser. Encyclopedia of mathematics and its applications. Cambridge ; New York: Cambridge University Press, 2006, no. 107, oCLC: ocm70059806.

[8] C. Bourlier, S. Bellez, H. Li, and G. Kubicke, "Sub-Domain Decomposition Iterative Method Combined With ACA: An Efficient Technique for the Scattering From a Large Highly Conducting Rough Sea Surface," IEEE Trans. Antennas Propagat., vol. 63, no. 2, pp. 659-666, Feb. 2015. [Online]. Available: http://ieeexplore.ieee.org/document/6965626/

[9] M. I. Mishchenko, Electromagnetic Scattering by Particles and Particle Groups: An Introduction. Cambridge University Press, 2014.

[10] _ _ " "Independent" and "dependent" scattering by particles in a multiparticle group," OSA Continuum, vol. 1, no. 1, p. 243, Sep. 2018. [Online]. Available: https://www.osapublishing.org/abstract.cfm?URI=osac$1-1-243$

[11] _ - "Multiple scattering, radiative transfer, and weak localization in discrete random media: Unified microphysical approach," Rev. Geophys., vol. 46, no. 2, p. RG2003, Apr. 2008. [Online]. Available: http://doi.wiley.com/10.1029/2007RG000230

[12] A. K. Heidinger, C. O'Dell, R. Bennartz, and T. Greenwald, "The Successive-Order-of-Interaction Radiative Transfer Model Part I: Model Development," J. Appl. Meteor. Climatol., vol. 45, no. 10, pp. 1388-1402, Oct. 2006. [Online]. Available: http://journals.ametsoc.org/doi/10.1175/JAM2387.1

[13] R. Burkholder and T. Lundin, "Forward-backward iterative physical optics algorithm for computing the RCS of open-ended cavities," IEEE Trans. Antennas Propagat., vol. 53, no. 2, pp. 793-799, Feb. 2005. [Online]. Available: http://ieeexplore.ieee.org/document/1391151/

[14] I. Gershenzon, Y. Brick, and A. Boag, "Shadow radiation iterative physical optics method for high-frequency scattering," IEEE Transactions on Antennas and Propagation, vol. 66, no. 2, pp. 871-883, 2018

[15] F. Obelleiro-Basteiro, J. Luis Rodriguez, and R. J. Burkholder, "An iterative physical optics approach for analyzing the electromagnetic scattering by large open-ended cavities," IEEE Transactions on Antennas and Propagation, vol. 43, no. 4, pp. 356-361, 1995.

[16] C. Brezinski, "Convergence acceleration during the 20th century," Journal of Computational and Applied Mathematics, p. 21, 2000.

[17] C. Brezinski and M. Redivo Zaglia, Extrapolation methods: theory and practice, ser. Studies in computational mathematics. Amsterdam ; New York : New York, N.Y., U.S.A: North-Holland ; Distributors for the U.S and Canada, Elsevier Science Pub. Co, 1991, no. 2.

[18] A. Quarteroni, R. Sacco, and F. Saleri, Numerical mathematics, ser Texts in applied mathematics. New York: Springer, 2000, no. 37.

[19] R. Eftekhar, H. Hu, and Y. Zheng, "Convergence acceleration in scattering series and seismic waveform inversion using nonlinear Shanks transformation," Geophysical Journal International, vol. 214, no. 3, pp. 1732-1743, Sep. 2018. [Online]. Available: https://academic.oup.com/gji/article/214/3/1732/5034517

[20] S. Singh and Heng-Ming Tai, "Convergence acceleration of method of moments solution for antenna and scattering problems," in 2008 World Automation Congress, 2008, pp. 1-5.

[21] Y. Boubendir, F. Ecevit, and F. Reitich, "Acceleration of an iterative method for the evaluation of high-frequency multiple scattering effects," SIAM Journal on Scientific Computing, vol. 39, p. B1130-B1155, 12 2017.

[22] O. P. Bruno and F. Reitich, "Numerical solution of diffraction problems: a method of variation of boundaries," J. Opt. Soc. Am. A, vol. 10, no. 6, p. 1168, Jun. 1993. [Online]. Available: https://www.osapublishing.org/abstract.cfm?URI=josaa-10-6-1168

[23] R. E. Kleinman, G. F. Roach, and P. M. van den Berg, "Convergent Born series for large refractive indices," J. Opt. Soc. Am. A, vol. 7, no. 5, p. 890, May 1990. [Online]. Available: https://www.osapublishing.org/abstract.cfm?URI=josaa-7-5-890

[24] A. Samokhin, Y. Shestopalov, and K. Kobayashi, "Stationary iteration methods for solving 3d electromagnetic scattering problems," Applied Mathematics and Computation, vol. 222, 092012.

[25] G. Osnabrugge, S. Leedumrongwatthanakun, and I. M. Vellekoop, "A convergent Born series for solving the inhomogeneous Helmholtz equation in arbitrarily large media," Journal of Computational Physics, vol. 322, pp. 113-124, Oct. 2016. [Online]. Available: https://linkinghub.elsevier.com/retrieve/pii/S0021999116302595
[26] S. Lang, Complex Analysis, ser. Graduate Texts in Mathematics. New York, NY: Springer New York, 1999, vol. 103. [Online]. Available: http://link.springer.com/10.1007/978-1-4757-3083-8

[27] R. E. Scraton, "The practical use of the Euler transformation," $B I T$, vol. 29 , no. 2, pp. 356-360, Jun. 1989. [Online]. Available: http://link.springer.com/10.1007/BF01952689

[28] Y. Saad, Iterative Methods for Sparse Linear Systems, 2nd ed. USA: Society for Industrial and Applied Mathematics, 2003.

[29] A. B. Thaheem and A. Laradji, "Classroom note: A generalization of Leibniz rule for higher derivatives," International Journal of Mathematical Education in Science and Technology, vol. 34, no. 6, pp. 905-907, 2003. [Online]. Available: https://doi.org/10.1080/00207390310001595410

[30] O. P. Bruno and F. Reitich, "Approximation of analytic functions: a method of enhanced convergence," Math. Comp. vol. 63, no. 207, pp. 195-195, Sep. 1994. [Online]. Available: http://www.ams.org/jourcgi/jour-getitem?pii=S0025-5718-19941240654-9

[31] K. F. Warnick, Numerical analysis for electromagnetic integral equations. Boston: Artech House, 2008, oCLC: ocn231588156.

[32] J. P. Boyd, "A proof, based on the euler sum acceleration, of the recovery of an exponential (geometric) rate of convergence for the fourier series of a function with gibbs phenomenon," in Spectral and High Order Methods for Partial Differential Equations, J. S. Hesthaven and E. M. Rønquist, Eds. Berlin, Heidelberg: Springer Berlin Heidelberg, 2011, pp. 131-139.

[33] S. Murashige and K. Tanaka, "A new method of convergence acceleration of series expansion for analytic functions in the complex domain," Japan J. Indust. Appl. Math., vol. 32, no. 1, pp. 95-117, Mar. 2015. [Online]. Available: http://link.springer.com/10.1007/s13160-0140159-z

[34] M. Abramowitz and I. A. Stegun, Handbook of Mathematical Functions with Formulas, Graphs, and Mathematical Tables, ninth dover printing, tenth gpo printing ed. New York: Dover, 1964

[35] K. Sertel and J. Volakis, Integral Equation Methods for Electromagnetics. Institution of Engineering and Technology, Jan. 2012. [Online] Available: https://digital-library.theiet.org/content/books/ew/sbew045e

[36] C. Balanis, Advanced Engineering Electromagnetics, 2nd Edition. New York: Wiley, 2012

[37] W. Gibson, The Method of Moments in Electromagnetics. CRC Press, 2014.

[38] G. Starke and R. S. Varga, "A hybrid Arnoldi-Faber iterative method for nonsymmetric systems of linear equations," Numer Math., vol. 64, no. 1, pp. 213-240, Dec. 1993. [Online]. Available: http://link.springer.com/10.1007/BF01388688

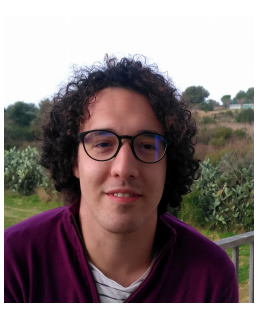

Hector Lopez-Menchon was born in Barcelona, Spain, in 1994. He received the bachelor's degree in Engineering Physics and the master's degree in Innovation and Research in Informatics from the Universitat Politècnica de Catalunya (UPC), Barcelona, in 2017 and 2019, respectively. He has hold the position of Research Support Personnel in the UPC and is currently pursuing the $\mathrm{PhD}$ degree at the Signal Theory and Communications Department in the same university.

He has authored articles in refereed international journals and international conferences. His research interests include computational electromagnetics, numerical linear algebra, high performance computing and parallel algorithms. 


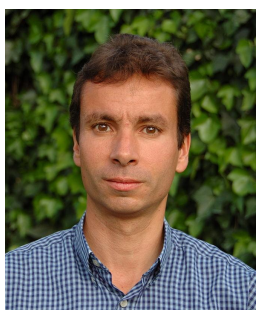

Juan M. Rius (S'88-M'91-SM'12) was Born in Barcelona, Spain, in 1963. He received the "Ingeniero de Telecomunicación" degree in 1987 and the "Doctor Ingeniero" degree in 1991, both from the Universitat Politècnica de Catalunya (UPC), Barcelona, Spain. In 1985 he joined the Electromagnetic and Photonic Engineering group at UPC, now the CommSensLab, where he currently holds a position of "Catedrático" (equivalent to Full Professor).

From 1985 to 1988 he developed a new inverse scattering algorithm for microwave tomography in cylindrical geometry systems. Since 1989 he has been engaged in the research for new and efficient methods for numerical computation of electromagnetic scattering and radiation. He is the developer of the Graphical Electromagnetic Computation (GRECO) approach for high-frequency RCS computation, the Integral Equation formulation of the Measured Equation of Invariance (IEMEI) and the Multilevel Matrix Decomposition Algorithm (MLMDA) in 3D. Current interests are the numerical simulation of electrically large antennas and scatterers.

$\mathrm{He}$ has held positions of "Visiting Professor" at EPFL (Lausanne) from May 1, 1996 to October 31, 1996; "Visiting Fellow" at City University of Hong Kong from January 3, 1997 to February 4, 1997; "CLUSTER chair" at EPFL from December 1, 1997 to January 31, 1998; and "Visiting Professor" at EPFL from April 1, 2001 to June 30, 2001.

He has more than 72 papers published or accepted in refereed international journals (43 in IEEE Trans.) and more than 200 in international conference proceedings.

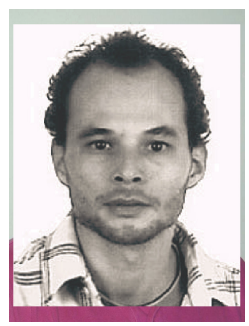

Alexander Heldring Alexander Heldring was born in Amsterdam, The Netherlands, in 1966. He received the M.S. degree in applied physics and the $\mathrm{Ph} . \mathrm{D}$. degree in electrical engineering from the Delft University of Technol- ogy, Delft, The Netherlands, in 1993 and 2002, respectively. He is currently an Associate Professor with the Telecommunications Department, Universitat Politécnica de Catalunya (UPC), Barcelona, Spain. He has authored over 30 articles in international journals and 100 in international conference pro- ceedings. His special research interest includes fast integral equation methods for electromagnetic problems.

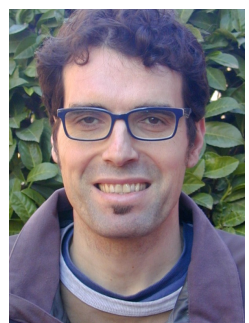

Eduard Ubeda Eduard Ubeda (Member, IEEE) was born in Barcelona, Spain, in 1971. He received the Telecom- munication Engineer and Doctor Ingeniero degrees from the Universitat Politècnica de Catalunya (UPC), Barcelona, in 1995 and 2001, respectively. In 1996, he was with the Joint Research Center from the European Commission, Ispra, Italy. From 1997 to 2000, he was a Research Assistant with the Electromagnetic and Photonic Engineering Group, UPC. From 2001 to 2002, he was a Visiting Scholar with the Electromagnetic Communication Labora- tory, Electrical Engineering Department, Pennsylvania State University (PSU), University Park, PA, USA. Since 2003, he has been with the Universitat Politècnica de Catalunya (UPC). He has authored over 30 articles in inter-national journals and 100 in international conference proceedings. His main research interests are numerical computation of scattering and radiation using integral-equation methods and advanced discretization techniques. 